\title{
Evaluating of a Super Bright LED as a Spectrophotometer Light Source at The Clinical Laboratory
}

\author{
Rangga Budi Santoso ${ }^{1}$, Dyah Titisari ${ }^{2}$, and M. Prastawa ATP ${ }^{1}$, Her Gumiwang ${ }^{1}$, Nora Bouzeghaia ${ }^{2}$ \\ Department of Electromedical Engineering Poltekkes Kemenkes, Surabaya, Indonesia \\ Department of Mechanical Engineering, Université Batna 2, Batna, Algeria
}

Corresponding author: Rangga Budi Santoso (p27838120117@gmail.com or ranggasantos17@gmail.com ).

This paragraph of the first footnote will contain support information, including sponsor and financial support acknowledgment.

\begin{abstract}
Spectrophotometers generally use a halogen lamp as a light source that passes through a filter (wavelength) according to the material to be analyzed. This study aims to analyze the ability of the LED as a light source on a spectrophotometer. In this study, the authors have determined blood sugar parameters as the test material. So that the determination of the wavelength of the LED as a light source must be adjusted to the specifications of the wavelength in the reagent manual procedure used. In the BAV Greiner Glucose Reagent procedure, the allowable wavelength is between $500-570 \mathrm{~nm}$ with a cuvette thickness of $1 \mathrm{~cm}$. They were measured against the reagent blank by the endpoint method. From this reference, the author uses an LED light source of $530 \mathrm{~nm}$, Epistar brand green. The module in this study consisted of a $530 \mathrm{~nm}$ LED lamp as a light source. Then a lens was added to focus the light beam from the $530 \mathrm{~nm}$ LED. The author also adds an aperture so that the light passing through is focused at one point of the circle. Further, the light will pass the cuvette. The results of the absorption of light will be received by the light sensor (photoresistor), and the data is processed by Arduino, and the results are displayed on display. From the results of this study, the value ranges error from $1 \%$ to $3 \%$ when a comparative test is carried out with the Analytical type Biolyzer 100 spectrophotometer with six different samples and is repeated five times each. From these data, it is found that the LED with a wavelength of $530 \mathrm{~nm}$ is effective as a light source for checking blood sugar.
\end{abstract}

INDEX TERMS 530 nm LED, Lens, Cuvette, Photoresistors, Arduino, Absorption, Reagent, Biolyzer 100

\section{INTRODUCTION}

Spectrophotometry is a method for measuring how much a chemical substance absorbs light by measuring the intensity of the light as a beam of light passes through a sample solution. The basic principle is that each compound absorbs or transmits light in a certain range of wavelengths. This measurement can also be used to measure the amount of a known chemical [1][2]. Spectrophotometry is a tool that relies on the quantitative analysis of molecules depending on how much light is absorbed by the colored compound. Important features of a spectrophotometer are the spectral bandwidth (range of colors that can be transmitted through the test sample), percentage of sample transmission, logarithmic range of sample absorption, and sometimes the percentage of reflectance measurements. Spectrophotometers are usually used to measure the transmittance or reflectance of solutions, transparent or opaque solids, such as polished glass or gases[3]. Although many biochemicals are colored, they absorb visible light and can therefore be measured by colorimetric procedures, and even colorless biochemicals can often be converted into colored compounds suitable for chromogenic coloration reactions to produce compounds suitable for colorimetric analysis [4]. The basic concept of a spectrophotometer is to use the Photometric Method. Beer Lambert's law states that the energy absorbed depends on the thickness of the material through which the light passes, and the amount of light absorbed (absorbed) is directly proportional to the concentration of the substance that absorbs or absorbs it 
[5][6]. In its application, spectrophotometry is widely used in laboratories, whether environmental laboratories, pharmacy laboratories, or health clinic laboratories. In the field of health clinical laboratories, a spectrophotometer or commonly known as a photometer is used to analyze the concentration of a solution from a patient sample using certain reagents according to the parameters to be analyzed, for example, blood sugar, uric acid, cholesterol, triglycerides, and so on. In general, spectrophotometry has important parts including, a lamp as a light source, a filter/wavelength selected according to the examination/measurement, and a light sensor as a receiver of the transmitted light [7]. Generally, spectrophotometers use halogen lamps as light sources with a lifetime of certain. In addition, there are filters/wavelengths that can be selected according to the desired examination [8][9][10]. Of course, such a spectrophotometric tool has a fairly high price, with a limited lifetime of halogen lamps. With the development of technology and knowledge, LEDs have various specifications, one of which is an LED that has a certain wavelength so that it can be selected according to needs [11][12][13]. The development of the era also makes it easier for us to know the amount of light intensity, with many choices of light sensors with certain types and specifications. Therefore, in this study, we will use LED as a spectrophotometer light source to analyze the solution according to the actual concentration.

In previous studies, many discussed LED Spectrophotometry with various methods, including the following. In 2015, Kim Ji Sun made a white LED spectrophotometer with a color sensor (TCS2300) as the receiver to analyze the color of a solution. As a result, the purity and wavelength can be detected with the CIE diagram, and the concentration can be estimated with the purity information. This method is more economical and efficient than the existing spectrophotometry [4]. Still, in the same year, Mohammad Karim Aly compared the LED spectrophotometer with a general spectrophotometer. The result was that the LED spectrophotometer had an error of $2.33 \%$, which means the results are acceptable [14]. The LED (white color) as a light source in a spectrophotometer was made by P. Visconti in 2017; the concept resembles a Spectro in general, only the light source is different. As a result, confirming the correct functioning and interaction of the system, via the PC terminal, between the user and the realized control unit [15]. In a Chaianantakul study, Natpasit made an LED-based spectrophotometer for blood sugar detection. As a result, the instrument showed good reproducibility and high sensitivity and specificity. Mini-spectrophotometers are promising to be used instead of conventional spectrophotometers for screening and monitoring glucose concentrations in patients with diabetes mellitus [16]. From the literature study above, it shows that the use of LEDs can be used as an alternative to a light source in a simple, economical spectrophotometer, but there is a need for studies or research that discusses the sensitivity and or effectiveness of light sensors in responding or accepting any existing conditions. In the literature above, it has not been explained in detail about the ability of the light sensor as an important element in determining the absorbance value of a solution being analyzed. In addition to the light sensor, the research method test results are still dominantly carried out on dyes whose concentrations are not stated in detail. Therefore, a study on LED analysis as a light source on a spectrophotometer needs to be carried out. In addition to the ability of the LEDs and light sensors to be analyzed, the test method will also use comparisons with parameters in clinical laboratories in hospitals that have calibration standards, QC (Quality Control) and reagent concentrations that have clear values. So it is hoped that it will provide detailed information

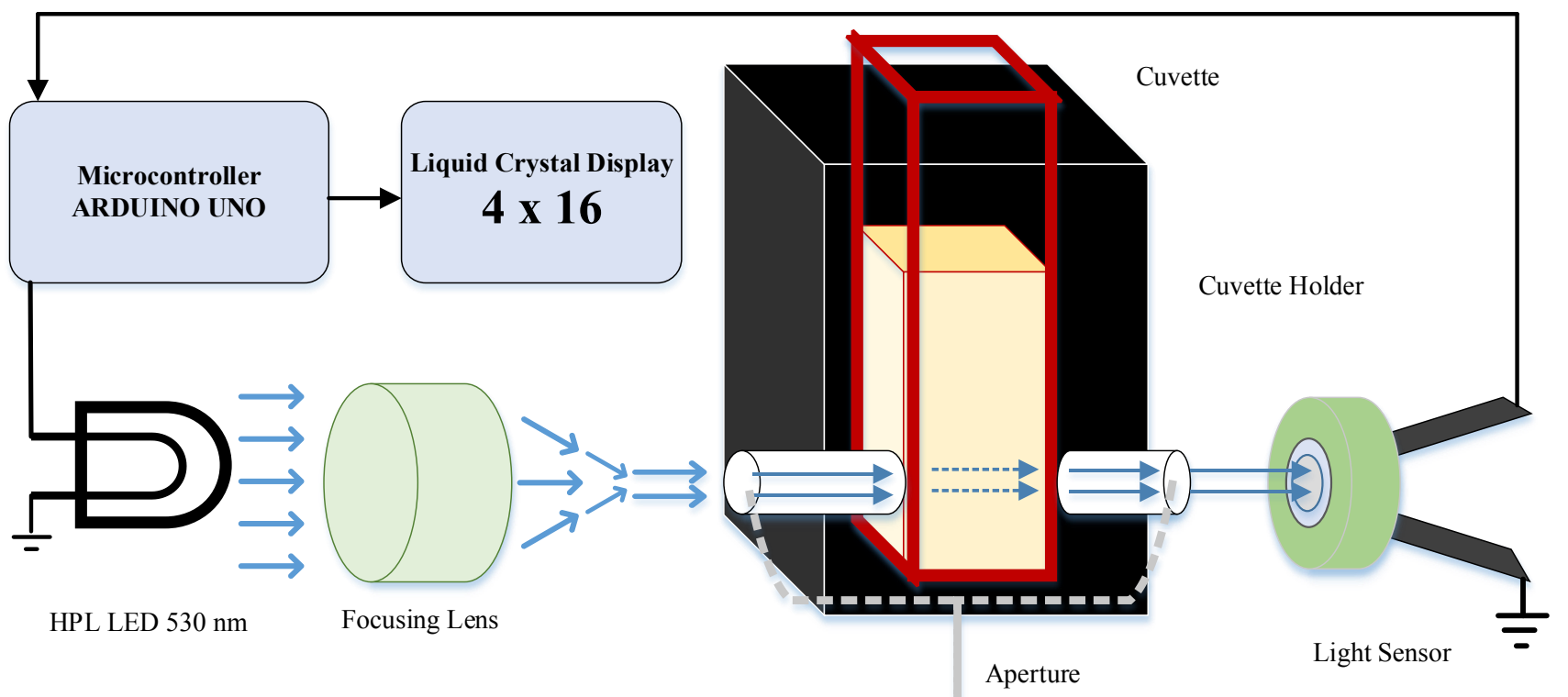

FIGURE 1. The diagram Block 
about the effectiveness of the LED's ability as a light source on a spectrophotometer.

\section{MATERIAL AND METHODS}

\section{A. EXPERIMENTAL SETUP}

In this study, the light sources used are LED HPL with Wavelength $530 \mathrm{~nm}$. The LED voltage input is given different conditions to get the best intensity. The light source will pass through the cuvette which has been filled with Glucose Working Reagent, which is reacted with the patient's sample (serum). Furthermore, the voltage value of the output on the light sensor is measured. The data analysis process is carried out by measuring the voltage value from the sensor output, then proceeding to analyze the results of the sample concentration, which is compared with the Analytical Biolyzer100 brand photometer.

\section{B. MATERIALS AND TOOL}

This module consists of LED with $530 \mathrm{~nm}$ as the light source, the lens to focus the light, cuvette, light sensor, Arduino Uno, and also the 4x16 LCD Display. Other materials there are Glucose Reagent, Calibrator/standard, Quality Control (QC), and the Analyzer Biolyzer100.

\section{EXPERIMENT}

In this study, an analysis will be carried out on the ability of the LED by giving different treatments on the LED input voltage. Each treatment will be used to perform Glucose Reagent with Quality Control (QC) as a validation Result whether each result is within QC range or not. The best result means the value is almost in the mean of the glucose value limit on the QC kit insert. So the best result will be used to analyze the blood sugar of the patient and will compare with the analyzer Biolyzer100.

\section{THE DIAGRAM BLOCK}

The light source uses an LED with a wavelength of $530 \mathrm{~nm}$ (FIGURE 1). Will be forwarded to a cuvette that has been filled by a mixture of working reagents with patient samples (serum). The absorbed light is then received by the light detector/sensor. The light sensor will respond in the form of analog data that is sent to the processor to be processed into digital data. The data is converted into absorbance values. Then it is changed again to the concentration value. The results of the concentration value will be shown on display. This research will begin with the design of an LED Spectrophotometer as a device for testing. Then the next research step is to measure the absorbance and concentration of the output readings of the two sensors that will be compared. The technical implementation is to change the sensor position alternately while still paying attention to the correct sensor position. The sample testing mechanism uses patient samples (serum), STD from BAV Greiner brand Glucose reagent, and Quality Control (Unitrol) BAV Greiner brand. Each of these samples will take data for comparison with the Analyticon brand Biolyzer 100 comparison tool, Germany. Glucose reagent is prepared for sample measurement. Read aquadest for initial standardization on unit tests. Then read the reagent blank (R1). This serves as the starting point for reading the Endpoint method. Then confirm whether it is necessary or not to do the standard. If the standard is done, the liquid that is inserted into the device is glucose reagent (R1) added to the standard. The reading results will be in the form of coefficient values, the data will be saved. Then proceed with the reading of the sample or QC (Quality Control). The result of reading the absorbance sample / QC is multiplied by the standard coefficient that has been formed earlier, and the result is the concentration value of the sample / QC.

\section{E. Analog}

The main circuit in this study has three circuit blocks: a light sensor circuit, a microcontroller circuit, and an LCD circuit. The light sensor circuit is a circuit that functions to receive light from the absorption of sample readings. The light sensor will detect changes in the light from the sample reading. The intensity received by the light sensor will be converted into resistance. Then there will be a voltage division. The voltage division will enter the Arduino A0 pin to be converted into ADC (Analog Digital Converter) data. This circuit has the characteristic that if the intensity received by the light sensor is large, the result of the voltage division will also be large. And vice versa, if the light sensor detects a low intensity, the resulting voltage will also be below.

Arduino circuit functions to process all information from the light sensor. In Arduino, the author has compiled a program starting from converting data from analog to digital data, and then the data is entered into the formula for calculating the absorbance value. After obtaining the absorbance value, it is continued with calculations to find the concentration of the readable solution. In addition, the author has also created a storage system to store absorbance data for blanks and factor values. Then the concentration results will be displayed on the LCD

\section{RESULT}

\section{A. MODUL DESIGN}

The final result of the block diagram is shown in FIGURE 2. An additional lens is used to focus the light and add an aperture before and after the cuvette. It is important to make the light beam pass the cuvette, and the sensor can detect well without any interferences. The final design, FIGURE 2, consists of 3 buttons, they are Yes, Next, and Reset. There is a display LCD with $4 \times 16$ and a hole to take the cuvette.

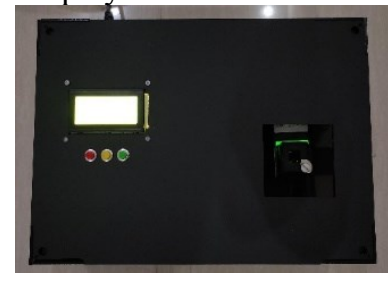

(a)

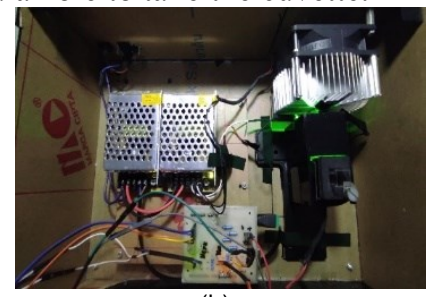

(b)
FIGURE 2. The spectrophotometer based on super bright LED (a) the panel side, (b) inside the module

\section{B. RESULT OF MEASUREMENT}

To analyze the capability of LED in this study to perform reading the absorbance of the glucose quality control and 
evaluate the result value, whether within range or not (TABLE 1).

TABLE 1

LED Test with Glucose Quality Control (QC)

\begin{tabular}{cccccc}
\hline TREATMENT & $\begin{array}{c}\text { LED } \\
\text { Conditi } \\
\text { on }\end{array}$ & $\begin{array}{c}\text { Sensor } \\
\text { Output } \\
\mathbf{( m V )}\end{array}$ & ABS & $\begin{array}{c}\text { QC } \\
(\mathbf{m g} / \mathbf{d l})\end{array}$ & $\begin{array}{c}\text { QC } \\
\text { Range } \\
\text { (mg/d) }\end{array}$ \\
\hline $\begin{array}{c}\text { Treatment 1 } \\
\text { Vin LED 5.9V }\end{array}$ & Dim & 0.475 & 0.029 & - & \\
$\begin{array}{c}\text { Treatment 2 } \\
\text { Vin LED 6.3 V }\end{array}$ & Medium & 0.625 & 0.276 & 120.1 & $\mathbf{7 5 . 9}$ \\
$\begin{array}{c}\text { Treatment 3 } \\
\text { Vin LED 6.5V }\end{array}$ & Bright & 0.692 & 0.216 & 89.2 & $\mathbf{1 0 3}$ \\
$\begin{array}{c}\text { Treatment 4 } \\
\text { Vin LED 12V }\end{array}$ & Bright & 0.855 & 0.208 & 85.2 & \\
\hline
\end{tabular}

Based on the results of Quality Control readings in Treatment 3 with a power range of 5 watts and Treatment 4 with a range of 10 watts of power indicates that the concentration is within the allowable limits. While in treatment 2 , with a power range of 4 watts, the concentration results were above the allowable limit. So that Treatments 3 and 4 can be used for testing blood sugar concentrations. However, the author uses Treatment 4 for testing blood sugar concentrations because it maximizes the ability of the LED to work optimally at a voltage of 12 Volts with a maximum power of 10 watts.

\section{MODULE COMPARISON WITH BIOLYZER100}

Data collection on the patient's blood sugar concentration in this study used random blood/serum samples. In addition, the blood sugar that is measured is random blood sugar (without fasting). The author took six samples of the patient's blood/serum, then reacted with reagents and read them in the module and also in the comparison device (Biolyzer100) at the same time. This is necessary and concern because the chemical reactions that occur between the reagents and the blood/serum samples will continue to run. So that the incubation time according to the reagent procedure needs to be considered, and the reading process in the module and comparison must be carried out simultaneously.

From the results of the measurements that have been carried out, it can be seen that the higher the absorbance value of the sample, the higher the concentration of the sample itself (fig. It can be seen from the comparative test that the author did as in the linear curve of the comparison of the concentration results with the sample absorbance on the module and comparison tool (Biolyzer100) as follows.

TABLE 2.

Reagent Blank and Factor Value

\begin{tabular}{ccc}
\hline Parameter Measure & Module & Biolyzer100 \\
\hline Reagent Blank & 0.076 & 0.091 \\
Factor & 301.205 & 346,021
\end{tabular}

TABLE 3

Measurement from Patients

\begin{tabular}{|c|c|c|c|c|c|}
\hline \multicolumn{6}{|c|}{ iveasurememiliom ratiems } \\
\hline \multirow{2}{*}{ PX } & \multicolumn{2}{|c|}{ BIOLYZER 100} & \multicolumn{2}{|c|}{ MODULE } & \multirow{2}{*}{ Error $\%$} \\
\hline & ABS & CONC. & ABS & CONC. & \\
\hline 1 & 0.248 & 85,808 & 0.293 & 88,279 & $3 \%$ \\
\hline 2 & 0.366 & 126.77 & 0.435 & 130,946 & $3 \%$ \\
\hline 3 & 0.697 & 242,902 & 0.799 & 240,690 & $1 \%$ \\
\hline 4 & 0.305 & 105.502 & 0.345 & 104.050 & $1 \%$ \\
\hline 5 & 0.381 & 131.754 & 0.431 & 128.768 & $2 \%$ \\
\hline 6 & 0.391 & 135.154 & 0.442 & 132.976 & $2 \%$ \\
\hline
\end{tabular}

Based on the linearity graph (FIGURE 3), there are two graphic results, namely the graph of the results of the comparison tool and the author's module. The result is the tool that the author made has a ratio of $\mathrm{R}^{2}=0.9999$. At the same time, the comparison device (Biolyzer100) has a value of $\mathrm{R}^{2}=1$. This means that the absorbance value is directly proportional to the concentration value. If the absorbance value is high, the concentration will also be high. Likewise, when compared with the Biolyzer 100 device, it has an Rvalue $=1$.

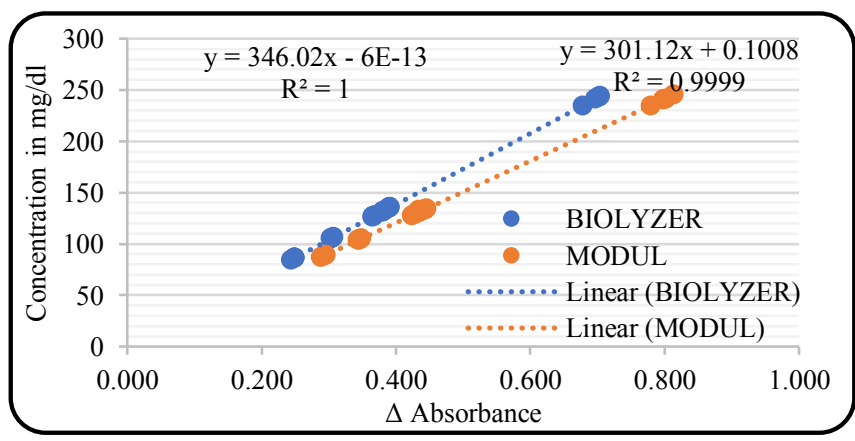

FIGURE 3. Linearity Graph of Absorbance and Concentration

\section{DISCUSSION}

When the author's module was tested and compared using a patient sample, the results showed any value error ranging from $1 \%-3 \%$. The Value error can occur due to several things. Among them are material conditions (reagents, QC, and standards), incubation time, and stability of absorbance readings on the module. In addition, there are some differences in the hardware of the writer's tool and the comparison tool, such as the wavelength used. In the module, an LED with a wavelength of $530 \mathrm{~nm}$ is used, while the comparison tool uses a Filter/Wavelength of $500 \mathrm{~nm}$. This is still allowed to be done referring to the procedure for using glucose reagents as in table 1 .

The mechanical design that the author made for this research is to use an LED with a wavelength of $530 \mathrm{~nm}$ as a 
light source that is directly forwarded and focused using a lens through a cuvette and detected by a light sensor. An LED with a wavelength of $530 \mathrm{~nm}$ has a green color. In contrast, the comparison device (Biolyzer100) uses a halogen lamp as a light source [32]. Also, equally, there is a lens that functions to focus the light from the halogen lamp, then it is forwarded to the cuvette and passed through a filter with a wavelength of $500 \mathrm{~nm}$, which is then detected by the light sensor. This difference resulted in one of the deviations errors in this study.

\section{CONCLUSION}

Based on the purpose of this research that the author wants to analyze the ability of LEDs and light sensors, to read glucose samples concentration. So the conclusions can be drawn. The selection of a green LED with a wavelength of $530 \mathrm{~nm}$ can be used as a spectrophotometer light source for checking blood sugar, and the power is within ten Watt. The addition of a lens serves to focus the LED light beam so that it can enter the slit, helping to optimize the light intensity from the LED to the sensor. From the test results using a reagent blood sugar, we get an error of $1.6 \%$ on the results of the Quality Control and 1-3\% in comparison with the results of the reading six patients looping five times respectively, with comparable commercial instrument Biolyzer 100.

In this study, there are still shortcomings, so improvements are needed. Among them, the need for sensor reading signal processing modules so that more stable data is obtained.

\section{REFERENCES}

[1] D. A. Pfeffer et al., "Quantification of glucose-6-phosphate dehydrogenase activity by spectrophotometry: A systematic review and meta-analysis," PLoS Med., vol. 17, no. 5, pp. 1-18, 2020, doi: 10.1371/journal.pmed.1003084.

[2] K. Mohammad, A. Zekry, and M. Abouelatta-Ebrahim, "LED Based Spectrophotometer can compete with conventional one," Int. J. Eng. Technol., vol. 4(2), May 2015, doi: 10.14419/ijet.v4i2.4504.

[3] R. Giovannetti, "The Use of Spectrophotometry UV-Vis for the Study of Porphyrins," Macro To Nano Spectrosc., 2012, doi: 10.5772/38797.

[4] J. S. Kim et al., "Simple LED spectrophotometer for analysis of color information," Biomed. Mater. Eng., vol. 26, pp. S1773-S1780, 2015, doi: 10.3233/BME-151478.

[5] S. Mandal and M. O. Manasreh, "An in-vitro optical sensor designed to estimate Glycated Hemoglobin Levels," Sensors (Switzerland), vol. 18, no. 4, 2018, doi: 10.3390/s18041084.

[6] P. J. Chen et al., "A practical portable photometer using LEDs as inspection light source," I2MTC 2017 - 2017 IEEE Int. Instrum. Meas. Technol. Conf. Proc., pp. 1-6, 2017, doi 10.1109/I2MTC.2017.7969714.

[7] D. R. Albert, M. A. Todt, and H. F. Davis, "A low-cost quantitative absorption spectrophotometer," J. Chem. Educ., vol. 89, no. 11, pp. 1432-1435, 2012, doi: 10.1021/ed200829d.

[8] K. M. Zielinska-Dabkowska, J. Hartmann, and C. Sigillo, "LED light sources and their complex set-up for visually and biologically effective illumination for ornamental indoor plants," Sustain., vol. 11, no. 9, 2019, doi: 10.3390/su11092642.

[9] C. A. De Caro, "UV / VIS Spectrophotometry," Mettler-Toledo Int., no. September 2015, pp. 4-14, 2015.

[10] M. A. Al-Dhaheri, N. E. Mekkakia-Maaza, H. Mouhadjer, and A. Lakhdari, "Noninvasive blood glucose monitoring system based on near-infrared method," Int. J. Electr. Comput. Eng., vol. 10, no. 2, pp.
1736-1746, 2020, doi: 10.11591/ijece.v10i2.pp1736-1746.

[11] T. Pulli, T. Dönsberg, T. Poikonen, F. Manoocheri, P. Kärhä, and E. Ikonen, "Advantages of white LED lamps and new detector technology in photometry," Light Sci. Appl., vol. 4, no. June, pp. 1-7, 2015, doi: 10.1038/lsa.2015.105.

[12] T. Dönsberg et al., "Methods for decreasing uncertainties in LED photometry," 17th Int. Congr. Metrol. CIM 2015, vol. 1, pp. 1-6, 2015, doi: $10.1051 /$ metrology/20150011001.

[13] A. B. D. Nandiyanto, R. Zaen, R. Oktiani, A. G. Abdullah, and L. S. Riza, "A simple, rapid analysis, portable, low-cost, and Arduino-based spectrophotometer with white LED as a light source for analyzing solution concentration," Telkomnika (Telecommunication Comput. Electron. Control., vol. 16, no. 2, pp. 580-585, 2018, doi: 10.12928/TELKOMNIKA.v16i2.7159.

[14] K. A. Mohammad, A. Zekry, and M. Abouelatta, "LED Based Spectrophotometer can compete with conventional one," Int. J. Eng. Technol., vol. 4, no. 2, p. 399, 2015, doi: 10.14419/ijet.v4i2.4504.

[15] P. Visconti, A. Lay-Ekuakille, P. Primiceri, G. Ciccarese, and R. De Fazio, "Hardware design and software development for a white LEDbased experimental spectrophotometer managed by a PIC-based control system," IEEE Sens. J., vol. 17, no. 8, pp. 2507-2515, 2017, doi: 10.1109/JSEN.2017.2669529.

[16] N. Chaianantakul et al., "Development of mini-spectrophotometer for determination of plasma glucose," Spectrochim. Acta - Part A Mol. Biomol. Spectrosc., vol. 204, pp. 670-676, 2018, doi: 10.1016/j.saa.2018.06.107.

[17] A. Watane, J. Chabowski-carpino, C. Science, and T. Instruments, "GOLD : Glucose Optical LED Detection," 2017.

[18] D. T. M. Dinh, V. A. Truong, A. N. P. Tran, H. X. Le, and H. T. T. Pham, Non-invasive Glucose Monitoring System Utilizing NearInfrared Technology, vol. 69. Springer Singapore, 2020.

[19] N. A. M. Aziz, N. Arsad, P. S. Menon, S. Shaari, Z. M. Yusof, and A R. Laili, "An assessment study of absorption effect: LED vs tungsten halogen lamp for noninvasive glucose detection," J. Innov. Opt. Health Sci., vol. 8, no. 2, 2015, doi: 10.1142/S1793545815500133.

[20] R. B. Dominguez, M. A. Orozco, G. Chávez, and A. Márquez-Lucero, "The evaluation of a low-cost colorimeter for glucose detection in salivary samples," Sensors (Switzerland), vol. 17, no. 11, pp. 19-21, 2017, doi: 10.3390/s17112495.

[21] P. Jain, R. Maddila, and A. M. Joshi, "A precise non-invasive blood glucose measurement system using NIR spectroscopy and Huber's regression model," Opt. Quantum Electron., vol. 51, no. 2, pp. 1-15, 2019, doi: 10.1007/s11082-019-1766-3.

[22] B. Javid, F. G. Faranak, and F. S. Zakeri, "Noninvasive optical diagnostic techniques for mobile blood glucose and bilirubin monitoring," J. Med. Signals Sens., vol. 8, no. 3, pp. 125-139, 2018 , doi: 10.4103/jmss.JMSS-8-18.

[23] C. Kalkbrenner, A. Van, T. Smith, and D. Charles, "Low-Cost Spectrophotometer," pp. 1-17.

[24] T. R. Dias and B. F. Reis, "A LED based photometer for solid phase photometry: Zinc determination in pharmaceutical preparation employing a multicommuted flow analysis approach," J. Braz. Chem. Soc., vol. 23 , no. 8, pp. 1515-1522, 2012, doi: 10.1590/S010350532012005000014

[25] S. Q. Jin, C. Huang, G. Xia, M. Y. Hu, and Z. J. Liu, "Bandwidth correction in the spectral measurement of light-emitting diodes," $J$ Opt. Soc. Am. A, vol. 34, no. 9, p. 1476, 2017, doi: 10.1364/josaa.34.001476

[26] D. González-Morales, A. Valencia, A. Díaz-Nuñez, M. FuentesEstrada, O. López-Santos, and O. García-Beltrán, "Development of a low-cost UV-Vis spectrophotometer and its application for the detection of mercuric ions assisted by chemosensors," Sensors (Switzerland), vol. 20, no. 3, 2020, doi: 10.3390/s20030906.

[27] N. Demitri and A. M. Zoubir, "Measuring Blood Glucose Concentrations in Photometric Glucometers Requiring Very Small Sample Volumes," IEEE Trans. Biomed. Eng., vol. 64, no. 1, pp. 28 39, 2017, doi: 10.1109/TBME.2016.2530021.

[28] V. R. Pereira and B. S. Hosker, "Low-cost $(<€ 5)$, open-source, potential alternative to commercial spectrophotometers," PLoS Biol., vol. 17, no. 6, pp. 1-8, 2019, doi: 10.1371/journal.pbio.3000321.

[29] A. Wego, "Accuracy simulation of an LED based spectrophotometer," Optik (Stuttg)., vol. 124, no. 7, pp. 644-649, 2013, doi: 
10.1016/j.ijleo.2012.01.005.

[30] M. W. Prairie, S. H. Frisbie, K. K. Rao, A. H. Saksri, S. Parbat, and E. $\mathrm{J}$. Mitchell, An accurate, precise, and affordable light emitting diode spectrophotometer for drinking water and other testing with limited resources, vol. 15, no. 1. 2020

[31] M.-E. Bouza, A. Nastou, C. Panigyraki, and C. Makedonas, "Introducing spectrophotometry in the school lab employing LEGO bricks and LEDs," Chem. Teach. Int., vol. 1, no. 1, pp. 1-8, 2019, doi: 10.1515/cti-2018-0012.

[32] D. S. R. . Rahayu, M. R. Mak'ruf, and S. Syaifudin, "Luxmeter Design with Proximity Sensor to Efficiently Test Light Intensity and Distance

\section{Attachment :}

1. Schematic : https://drive.google.com/file/d/1yKLUkU7bIayQ4QRUVr-c7PnvJU7gviS/view?usp=sharing (File from Eagle)

2. Software :

https://drive.google.com/file/d/1yXbrnGk_OrPeV6UpcAadY ici_yCdkRxa/view?usp=sharing on Lamp Operation in Hospitals", International Journal of Advanced Health Science and Technology, vol. 1, no. 1, pp. 20-25, Oct. 2021. 